\title{
Anesthesia Concerns in Child with Takayasu Arteritis for Catheterization Laboratory Intervention
}

\author{
Kalpana Irpachi ${ }^{1} \quad$ Sandeep Sharan ${ }^{1} \quad$ Ameya Karanjkar \\ ${ }^{1}$ Department of Cardiac Anaesthesia, CT Center, All India Institute \\ of Medical Sciences, New Delhi
}

\author{
Poonam Malhotra Kapoor ${ }^{1}$
}

J Card Crit Care TSS 2020;3:49-50
Abstract
Keywords
- anesthesia concerns
- catheterization labora- tory intervention
- Takayasu's arteritis

\author{
Address for correspondence Kalpana Irpachi, MBBS, MD, DM \\ Department of Cardiac Anaesthesia, CT Center, All India Institute of \\ Medical Sciences, New Delhi, India (e-mail: drkalpna80@rediffmail.com).
}

\section{Introduction}

Takayasu's arteritis (TA) (also called the pulseless disease) is a nonspecific chronic progressive panendarteritis affecting the large- and medium-sized arteries, involving the aorta and its main branches. Anesthesia for patients with TA is complicated by severe uncontrolled hypertension, end-organ dysfunction, stenosis of major blood vessels, and difficulties encountered in monitoring arterial blood pressure. We describe the anesthetic concerns of a child with TA and severe hypertension refractory to medical treatment, presented with bilateral renal artery stenosis requiring urgent bilateral percutaneous renal angioplasty.

\section{Anesthesia Concerns}

TA is characterized by a focal stenosis process involving the aorta and the proximal segment of its major branches. In 1908, TA was first reported by Takayasu, a Japanese ophthalmologist; it then has subsequently been reported throughout the world. ${ }^{1}$

TA presents either incidentally or more frequently for correction of other vascular occlusive disease, often presenting as upper extremity claudication and minimal to no palpable arterial pulsations in the arms as evident in our patient. ${ }^{2}$

We encountered a 6 -year-old boy weighing $13 \mathrm{~kg}$ who presented with breathlessness, hypertension refractory to medical treatment, and bilateral subclavian and bilateral renal artery stenosis; he was subsequently found to have TA with severe eccentric mitral regurgitation due to mitral valve prolapse with pulmoary artery hypertension (PAH) requiring urgent bilateral percutaneous renal angioplasty (PTRA). Most of these procedures are generally performed under general anesthesia or local anesthesia in older children. There are certain advantages and disadvantages of both general as well as regional anesthesia. Administering general anesthesia is challenging in TA as it needs special attention for major vascular procedures. ${ }^{3}$ The influence of changing head position on cerebral function should be evaluated because hyperextension of head during laryngoscopy may further compromise blood flow through carotid artery as a result of the vascular inflammatory process associated with this disease. Gaba et $\mathrm{al}^{4}$ suggested blind insertion of Intubating Laryngeal Mask Airway (ILMA) can be done with neck in the neutral position without any significant variation in degree of flexion or extension of the cervical spine during intubation.

Since the patient had pulmonary edema, he could not lie down supine, despite anticongestive therapy. The patient was planned for PTRA, under standard monitoring $\left(\mathrm{SpO}_{2}\right.$, noninvasive blood pressure, ECG, and temperature); the patient was sedated with bolus dose of fentanyl $2 \mu \mathrm{g} / \mathrm{kg}$ and ketamine $2 \mathrm{mg} / \mathrm{kg}$. The patient was maintained with $50 \%$ mixture of air and oxygen, and continuous titrated infusion was initiated with a combination of fentanyl
DOI https://doi.org/ $10.1055 / \mathrm{s}-0039-1696908$ ISSN 2457-0206.
Copyright @2020 Official

Publication of The Simulation

Society (TSS), accredited

by International Society of

Cardiovascular Ultrasound (ISCU).
License terms

(웅 (1) $\Theta$ 
$2 \mu \mathrm{g} / \mathrm{mL}(2-3 \mu \mathrm{g} / \mathrm{kg} / \mathrm{min})$, ketamine (10-15 $\mu \mathrm{g} / \mathrm{kg} / \mathrm{min})$, and midazolam $(1-2 \mu \mathrm{g} / \mathrm{kg} / \mathrm{min})$, respectively, at $12 \mathrm{~mL} / \mathrm{h}$ for sedation and pain control.

Musculoskeletal changes associated with TA make regional anesthesia technically more difficult. But the major advantage of regional anesthesia in such patients is that it allows monitoring of brain perfusion through the patient's level of consciousness, which is lost in general anesthesia.

In two studies, PTRA was performed under general anesthesia in children younger than 7 years and in older children, the procedure was performed under local anesthesia., 5

Two retrospective reviews of data for all children with renovascular hypertension who underwent PTRA, both at a single center between 1984 and 2003 and another between 1997 and 2009 were performed. All procedures were performed under general anesthesia. ${ }^{78}$ In 2010, Gumus et $\mathrm{al}^{9}$ reported PTRA in a 5-year-old child under continuous sedation as we performed successfully under monitored anesthesia care with continuous sedation in our patient. Certainly, if general anesthesia or intravenous sedation is used, neurologic monitoring is recommended if the patient has a history of syncope and carotid artery stenosis. The use of a computerized electroencephalography monitor during anesthesia allows continuous assessment of the adequacy of cerebral flow as we did the same in our patient.

Anesthetizing a patient with TA is a challenge as it is complicated by the four most important complications of TA-retinopathy, secondary uncontrolled hypertension, aortic regurgitation, and aneurysm formation, each graded as mild, moderate, or severe defined by Ishikawa. ${ }^{10}$ Cannulation of an artery for direct measurement of blood pressure carries an added risk for patients as the intimal changes in the vessels indicate the possibility of aneurysm formation. The main concern in conduct of anesthesia in patients with $\mathrm{TA}$ is the maintenance of blood pressure during the perioperative period.

A sodium nitroprusside infusion was continued at a rate of $2 \mu \mathrm{g} / \mathrm{kg} / \mathrm{min}$ during the procedure as well as in the postprocedure period for control of blood pressure. The therapeutic intervention period was uneventful. Arterial blood gas analyses were within normal limits.

Wang et $\mathrm{al}^{11}$ discussed the possible reasons for failure to resuscitate the patient. Failure of resuscitation may be due to poor myocardial performance and low cerebrovascular perfusion pressure. Anesthetic-induced decreased blood pressure caused by decreased cardiac output or systemic vascular resistance must be recognized promptly and treated by either adjusting the concentration of anesthetic drugs or expanding the intravascular fluid volume, or both. Post procedure, the child was monitored in the high dependency unit and had an unremarkable stay.

\section{Conclusion}

We recommend the meticulous preoperative planning of child presenting with TA before taking up for catheterization laboratory intervention, including need for preoperative optimization of the patient, periprocedural monitoring of the cardiovascular system, and maintenance of cerebral and other organ perfusion and normal acid-base status. The choice of the anesthesia technique should be tailored to the presentation of patients. Monitored anesthesia care using sedation for major vascular procedures and adequate preloading can be safely performed in a child presenting with TA. The goal should be maintenance of mean arterial pressure close to preoperative value to achieve favorable outcome. The role of anesthesiologist has become more challenging in this changing environment. Good coordination between anesthesiologist, cardiologist and cardiothoracic surgeon reduces the morbidity and mortality.

\section{Conflict of Interest}

None.

\section{References}

1 Takayasu M. Case with unusual changes of the vessels in the retina. Acta Soc Opthalmol Jpn 1908;12:554-555

2 Maffei S, Di Renzo M, Bova G, Auteri A, Pasqui AL. Takayasu's arteritis: a review of the literature. Intern Emerg Med 2006;1(2):105-112

3 Elarief M, Magadi P, Anaesthesia and pulseless disease case report and review of the literature. The Internet Journal of Anaesthesiology; 2004 10(1):...

4 Gaba P, Saxena KN, Dua CK. Takayasu's arteritis: anaesthetic implications and role of ILMA for airway management. Indian J Anaesth 2008;52:858

5 Zhu G, He F, Gu Y, et al. Angioplasty for pediatric renovascular hypertension: a 13-year experience. Diagn Interv Radiol 2014;20(3):285-292

6 Tullus, K, Brennan E, Hamilton G, Lord R, McLaren CA, Marks $\mathrm{SD}$, et al. Renovascular hypertension in children Lancet; 2008 371(9622):1453-1463

7 Shroff R, Roebuck DJ, Gordon I, et al. Angioplasty for renovascular hypertension in children: 20 -year experience. Pediatrics 2006;118(1):268-275

8 Srinivasan A, Krishnamurthy G, Fontalvo-Herazo L, et al. Angioplasty for renal artery stenosis in pediatric patients: an 11-year retrospective experience. J Vasc Interv Radiol 2010;21(11):1672-1680

9 Gumus B, Cevik H, Vuran C, Omay O, Kocyigit OI, Turkoz R. Cutting balloon angioplasty of bilateral renal artery stenosis due to Takayasu arteritis in a 5-year-old child with midterm follow-up. Cardiovasc Intervent Radiol 2010;33(2):394-397

10 Ishikawa K. Natural history and classification of occlusive thromboaortopathy (Takayasu's disease) Circulation 1978;57(1):27-35

11 Wang P-K, Luo B-H, Chen A, Lee Yi, Hsien-Yong Lai. Anesthetic considerations in Takayasu's arteritis-a case report. Tzu Chi Med J 2006;18:53-56 